\title{
Damping and higher multipole effects in the quantum electrodynamical model for electronic energy transfer in the condensed phase
}

\author{
Gregory D. Scholes ${ }^{a)}$ \\ Department of Chemistry, Imperial College of Science, Technology and Medicine, London SW7 2AY, \\ United Kingdom \\ David L. Andrews ${ }^{\text {b) }}$ \\ School of Chemical Sciences, University of East Anglia, Norwich NR4 7TJ, United Kingdom
}

(Received 5 December 1996; accepted 30 June 1997)

\begin{abstract}
The interplay between electronic coupling, spectral linewidth, and rate of electronic energy transfer between chromophores is examined in the context of a quantum electrodynamical (QED) model. The QED framework properly allows us to identify the partitioning between the near and far zone mechanisms for transfer of energy between chromophores dispersed in condensed phase (liquid or solid) host media. The extent to which coupling is modified by the medium is investigated. A general QED treatment of higher multipole contributions to the coupling between transition moments is also derived, whence interactions involving electric dipole, quadrupole and octopole as well as magnetic dipole and quadrupole interactions are examined explicitly. A new formulation is presented wherein expressions for the multipolar coupling tensors are obtained in terms of spherical Bessel functions, providing a clear, compact representation of the retarded coupling interaction and its distance-dependence. The irreducible tensor formulation of the coupling is discussed, highlighting features concerning the exact form of the orientation factors that have often in the past escaped notice. The detailed method of implementing a rotational averaging of the resultant interaction tensors is demonstrated, finally leading to a novel and concise representation for multipolar couplings of arbitrary order. The coupling between bacteriochlorophyll $a$ chromophores is discussed as an example. (C) 1997 American Institute of Physics. [S0021-9606(97)02837-7]
\end{abstract}

\section{INTRODUCTION}

Electronic energy transfer (EET) is the process by means of which a chromophore remote from another initiallyexcited chromophore may indirectly be excited. ${ }^{1-7}$ (Here we use the term "chromophore" in the broadest sense, to denote any molecule, atom or ion, or any part of such with a distinct electronic identity and optical response.) It is known that EET plays an important function in light harvesting, solar energy conversion and photosynthesis-it also significantly contributes to the operating characteristics of solid-state laser media. However, at the present time, further theoretical considerations of the mechanism of this fundamental phenomenon are still required in order to adduce the reasons underlying fast EET and energy migration observed in experiment. $^{8-11}$

If both chromophores, donor and acceptor, have allowed singlet-singlet transitions then the EET is promoted primarily by a coupling (approximately dipole-dipole) between transition moments. ${ }^{6,12,13}$ The nature of such an interaction has been examined previously within the framework of quantum electrodynamics, and two principal interaction regimes have been established. ${ }^{14-22}$ At close separations (the near zone) the familiar $R^{-6}$ distance dependence for the rate of (dipole-dipole) EET is recovered. At large separations, where the separation between donor and acceptor chromophores exceeds the appropriate reduced wavelength of

\footnotetext{
${ }^{a)}$ Electronic mail: g.scholes@ic.ac.uk

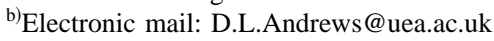

light (the far zone), a trivial radiative EET mechanism is found to operate. This involves emission and absorption of a real photon and leads to an $R^{-2}$ distance dependence for the rate of EET. Over intermediate distances, the energy transfer mechanism has a more intricate $R$-dependence.

In the present work the electronic coupling-linewidth relationship (noted previously ${ }^{23}$ as a factor determining the "adiabaticity" of a vibronic interaction) is investigated by incorporating line broadening into the quantum electrodynamical model for EET. As recent nonlinear spectroscopic studies of condensed phase photophysics show, ${ }^{24}$ the interplay between electronic processes and random bath fluctuations is critical in determining the rate of transition. The quantum electrodynamical framework affords a general and lucid formulation, and permits a more appropriate partitioning between near and far zone mechanisms for molecular systems in the condensed phase. We address the question: Is the electronic coupling modified significantly the solvent/ host medium?

In Sec. III a QED treatment of higher multipole contributions to the general retarded coupling between transition moments is presented. Thence interactions involving electric dipole, quadrupole, and octopole as well as magnetic dipole and quadrupole interactions are examined. A new formulation is derived, which obtains a general, limpid representation of the retarded coupling and its distance-dependence. We investigate the form of these interactions with particular regard to their irreducible tensorial structure, highlighting the complete form of the interaction, including phase factors. 

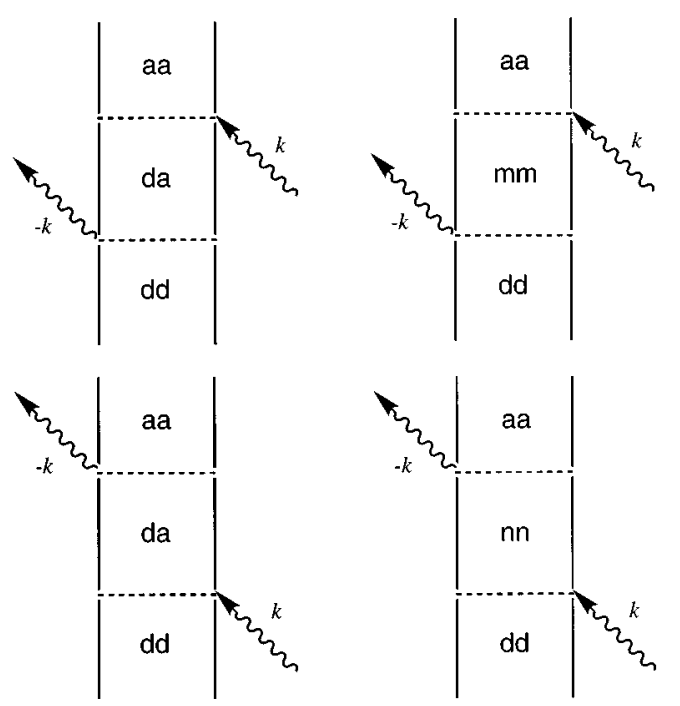

FIG. 1. Double-sided Feynman diagrams for electromagnetic field-mediated energy transfer involving virtual photon emission-absorption (upper diagrams) or absorption-emission (lower diagrams) interactions.

Appropriate expressions for the requisite rotational averages are formulated, leading to a concise representation for multipolar couplings of arbitrary order. The near and wave zone regime limits are compared and discussed.

\section{A QED FRAMEWORK FOR THE CONDENSED PHASE}

\section{A. Rate expressions}

In this section it is demonstrated how the framework previously established for the molecular quantum electrodynamical (QED) treatment of EET (Refs. 15-21) maybe combined with a density matrix-based treatment of spectral line shape accommodating multiphoton processes. ${ }^{25-27}$ Such a unification is symbiotic. For instance, homogeneously and inhomogeneously-broadened transition line shapes are incorporated into the QED formalism (e.g., by using a Brownian oscillator model for the molecule-bath coupling ${ }^{27}$ ), permitting meaningful extrapolation between the weak and strong coupling limits. At the same time, microscopic radiative interactions are properly introduced into the Liouville space model. We begin by discussing rate expressions, then we consider the coupling between transition moments.

Recently Juzeliūnas and Andrews have accommodated medium effects in a quantum electrodynamical treatment of resonant energy transfer in condensed matter. ${ }^{28,29}$ In the present work we wish to extend this work by examining damping effects in relation to the dynamic line-broadening caused by coupling with the host or solvent. We adopt the stochastic approach used previously for the description of absorption line shapes, ${ }^{25,30}$ resonance Raman and fluorescence emission phenomena ${ }^{31}$ and multiphoton processes, ${ }^{26,27,32-34}$ allowing elucidation of line broadening effects without recourse to microscopic detail.

We consider explicitly here the Liouville space pathways for interactions between the donor molecule $D$ and the acceptor $A$, as depicted in Fig. 1. The initial state $|d\rangle$ corresponds to excited $D$, ground state $A$, and a vacuum radiation field, $\left|D^{*} A\left(Q_{t}\right) ; 0\right\rangle$. The initial molecule and solvent coordinates $\left(Q_{0}\right)$ correspond to those of the ground state, $\left|D A\left(Q_{0}\right) ; 0\right\rangle$. At time $t$ these coordinates are written $Q_{t}$. The $Q$ represent the vibrational component of the propagation of a wave packet along the reaction coordinate so as to provide, for example, a Stokes' shift of the donor excitation energy. ${ }^{35,36}$ The final state $|a\rangle$ denotes $\left|D A^{*}\left(Q_{t}\right) ; 0\right\rangle$ and the intermediate states $|m\rangle$ and $|n\rangle$ include the configurations $\left|D A\left(Q_{t}\right) ; 1(\mathbf{k}, \lambda)\right\rangle$ and $\left|D^{*} A^{*}\left(Q_{t}\right) ; 1(\mathbf{k}, \lambda)\right\rangle$, respectively, where $1(\mathbf{k}, \lambda)$ denotes a single quantum excitation of the radiation field characterized by wave vector $\mathbf{k}$ and polarization $\lambda$.

The Hamiltonian is written $H=H_{0}+V$, with

$$
\begin{aligned}
H_{0}=\mid & d\rangle\left[E_{r 0}\left(Q_{0}\right)+g_{d}(t)\right]\langle d|+| a\rangle\left[E_{s 0}\left(Q_{0}\right)+g_{a}(t)\right] \\
& \times\langle a|+| m\rangle\left[\hbar c k+g_{m}(t)\right]\langle m|+| n\rangle \\
& \times\left[E_{r 0}\left(Q_{t}\right)+E_{s 0}\left(Q_{t}\right)+\hbar c k+g_{n}(t)\right]\langle n|,
\end{aligned}
$$

where $E_{i 0}\left(Q_{0}\right) \equiv E_{i}-E_{0}$ is the excitation energy from ground to excited state $i$ ( $i=r$ or $s$ for $D$ or $A$, respectively) at the ground state nuclear configuration $Q_{0}$. Equation (1) includes the Hamiltonian for the radiation field, expressed in terms of the transverse electric displacement vector $\mathbf{d}^{\perp}$ and fundamental magnetic field $\mathbf{b}$, integration being carried out over all space $\mathbf{r}$ ( $c$ is the speed of light in vacuo and $\epsilon_{0}$ is the vacuum permittivity). The $g_{i}(t)(i=d, a, n$ or $m)$ provide (possibly stochastic) modulations to the excitation and emission energies, originating from interactions between the chromophores and fluctuations of the bath. They would therefore be slow in a glassy or crystalline host, but fast in room temperature liquids (providing in the latter case an optical dephasing time that can be as short as a few tens of femtoseconds).

The interaction Hamiltonian is taken to be

$$
\begin{aligned}
V= & \sum_{m}\left(V_{d m}|d\rangle\left\langle m\left|+V_{m d}\right| m\right\rangle\left\langle d\left|+V_{a m}\right| a\right\rangle\right. \\
& \left.\times\left\langle m\left|+V_{m a}\right| m\right\rangle\langle a|\right),
\end{aligned}
$$

and the intermediate states are depicted in Fig. 2. For diagram (i) we have

$$
\begin{aligned}
V_{d m} & =\left\langle 1(\mathbf{k}, \lambda) ; E_{0}^{A}(Q), E_{0}^{D}(Q)\left|H_{\mathrm{int}}\right| E_{r}^{D}(Q), E_{0}^{A}(Q) ; 0\right\rangle \\
& =i \sum_{k, \lambda}\left(\frac{\hbar c k}{2 \epsilon_{0} V}\right)^{1 / 2} \bar{e}_{i}^{(\lambda)}(\mathbf{k}) \mu_{i}^{0 r}\left(Q_{D}\right) e^{-i \mathbf{k} \cdot \mathbf{R}_{D},} \\
V_{a m} & =\left\langle 0 ; E_{s}^{A}(Q), E_{0}^{D}(Q)\left|H_{\mathrm{int}}\right| E_{0}^{D}(Q), E_{0}^{A}(Q) ; 1(\mathbf{k}, \lambda)\right\rangle \\
& =-i \sum_{k, \lambda}\left(\frac{\hbar c k}{2 \epsilon_{0} V}\right)^{1 / 2} e_{j}^{(\lambda)}(\mathbf{k}) \mu_{j}^{s 0}\left(Q_{A}\right) e^{i \mathbf{k} \cdot \mathbf{R}_{A},}
\end{aligned}
$$

with

$$
H_{\mathrm{int}}=\sum_{N}-\epsilon_{0}^{-1} \mu\left(Q_{N}\right) \cdot \mathbf{d}^{\perp}\left(\mathbf{R}_{N}\right)
$$




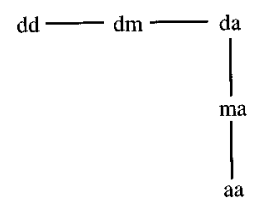

$K_{\mathrm{I}}$

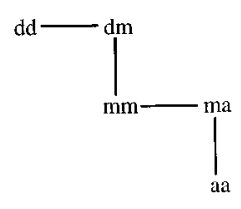

$K_{\mathrm{II}}$

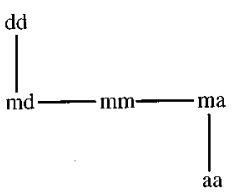

$K_{\mathrm{II}}$
FIG. 2. Liouville space pathways for the interaction contributions $K_{\mathrm{I}}, K_{\mathrm{II}}$, and $K_{\mathrm{III}}$.

for radiation modes characterized by polarization vectors $e_{i}^{(\lambda)}$ (an overbar denoting complex conjugation) with $V$ the quantization volume and $\mathbf{R}_{N}$ the position vector of molecule $N$. The operator $H_{\text {int }}$ is the radiation-matter interaction Hamiltonian, expressed as in Eq. (4), where $\mu\left(Q_{N}\right)$ is the dipole operator for molecule $N$ with nuclear coordinates $Q_{N}$ - at this stage we employ the dipole approximation to be consistent with previous work. This assumes that those interactions mediated via interchromophore orbital overlap are negligible, which should be the case for chromophores with quite strongly allowed electronic transitions, at intermediate to long separations, ${ }^{12,37}$ or for the coupling involving a carotenoid $S_{1}$ state, investigated recently. ${ }^{38}$ Interchromophore orbital overlap effects are ignored in this work, but higher multipole contributions to $H_{\text {int }}$ are considered in Sec. III. At close separations, where strong coupling obtains, a simple Golden Rule formulation of the rate probably no longer applies anyway; ${ }^{19,35}$ then the nature of the initially prepared state as well as electronic and vibrational coherences becomes important.

A sum over the appropriate manifold of vibrational states is associated with the transition moments $\mu_{i}^{0 r}$ and $\mu_{j}^{s 0}$, and it is assumed that $\mu_{i}$ is approximately independent of nuclear and solvent configuration. By expanding $Q_{N}$ about the equilibrium nuclear configuration in a Taylor expansion the Condon approximation may be applied and vibronic coupling may be included in Eqs. (3). ${ }^{23}$

In the usual QED treatment of EET we would now determine matrix elements for diagrams (i) and (ii) using second order perturbation theory (cf. Sec. III). Under appropriate conditions of weak coupling a rate may then be obtained using Fermi's Golden Rule. In this section, however, we will examine a generalized rate equation for EET via the Liouville equation for the density matrix, $\rho .^{27,39,40} \mathrm{We}$ may then obtain the generalized master equations of Eq. (5) (with the initial diagonality condition ${ }^{39}$ ) for the occupation probability of state $a, P_{a}(t) \equiv\langle a|\rho(t)| a\rangle$, where $\rho(t)$ is the density operator $\rho(t) \equiv|\Psi(t)\rangle\langle\Psi(t)|$ related to the wave function for the system $\Psi(t)$,

$$
\begin{gathered}
\frac{d P_{a}(t)}{d t}=\int_{0}^{t} d \tau \sum_{d}\left[W_{a d}(t-\tau) P_{d}(\tau)\right. \\
\left.-W_{d a}(t-\tau) P_{a}(\tau)\right], \\
\dot{\mathbf{P}}=\int_{0}^{t} d \tau \mathbf{W}(t-\tau) \mathbf{P}(\tau) .
\end{gathered}
$$

Equation (5b) is a more general formulation of Eq. (5a) involving the population vector $\mathbf{P}$ and the generalized rate matrix W.

By employing the time-ordered molecular propagator we obtain the tetradic generalized rate of Eq. (6) in terms of the Liouville space pathways depicted in Fig. 1. This explicitly incorporates the radiation field into the rate expression via the intermediate states $m$. The photon associated with $m$ may assume real or virtual character, essentially depending on its propagation time, and it will be shown here how this affects the dynamics of $\mathbf{P}$ [Eq. (5)]. For an intermediate state involving a real photon, there exists the possibility of a trivial energy transfer mechanism reflecting a classical sequence of emission and absorption events (as has been recognized previously ${ }^{18}$ ). It is possible to isolate from the population pathway, involving a real photon intermediate state, this trivial mechanism of the Bloch limit. It can be shown that the coherent pathway is given by the term $K_{\mathrm{I}}$ of Eq. (8), whilst the population pathway is given by $K_{\mathrm{II}}+K_{\mathrm{III}}$ in analogy to resonance Raman vs fluorescence, although we do not reproduce the derivation here.

Fluctuations in the transition frequencies are described in terms of an electronic frequency correlation function $M(t)$, using the line broadening function, $g_{i}(t)$, for a mode $i$ such that ${ }^{27,34}$

$$
g_{i}(\tau)=i \lambda_{i} \int_{0}^{t} d t_{1} M_{i}\left(t_{1}\right)+\left\langle\Delta \omega_{i}^{2}\right\rangle \int_{0}^{t} d t_{1} \int_{0}^{t_{1}} d t_{2} M_{i}\left(t_{2}\right) .
$$

This formulation thereby incorporates the reorganization energy $\lambda_{i}$ (providing the solvent Stokes' shift) and the coupling strength $\left\langle\Delta \omega_{i}^{2}\right\rangle^{1 / 2}$, which are related by the fluctuationdissipation theorem. We then have $g(t)=\Sigma_{i} g_{i}(t)$. The form of $g(t)$ dictates how frequency fluctuations are treated. ${ }^{27}$

There are six contributions to the memory kernel, which may be represented as the three Liouville space pathways of Fig. 1 and their complex conjugates. These are given as

$$
\begin{aligned}
K_{\mathrm{I}}(\omega)= & \operatorname{Re} \sum_{m, m^{\prime}}(-i)\left\langle V_{m^{\prime} d} G_{m^{\prime} a}^{\dagger}\left(\omega_{3}\right) V_{a m^{\prime}} G_{d a}\left(\omega_{2}\right)\right. \\
& \left.\times V_{m a} G_{d m}\left(\omega_{1}\right) V_{d m} \rho_{d}+\text { c.c. }\right\rangle, \\
K_{\mathrm{II}}(\omega)= & \operatorname{Re} \sum_{m, m^{\prime}}(-i)\left\langle V_{m^{\prime} d}\left(\omega_{3}\right) G_{m^{\prime} a} V_{a m^{\prime}} G_{m^{\prime} m}^{\dagger}\left(\omega_{2}\right)\right. \\
& \left.\times V_{m a} G_{d m}\left(\omega_{1}\right) V_{d m} \rho_{d}+\text { c.c. }\right\rangle, \\
K_{\mathrm{III}}(\omega)= & \operatorname{Re} \sum_{m, m^{\prime}}(-i)\left\langle V_{m^{\prime} d}\left(\omega_{3}\right) G_{m^{\prime} a}^{\dagger} V_{a m^{\prime}} G_{m^{\prime} m}^{\dagger}\left(\omega_{2}\right)\right. \\
& \left.\times V_{m a} G_{m^{\prime} d}\left(\omega_{1}\right) V_{d m} \rho_{d}+\text { c.c. }\right\rangle,
\end{aligned}
$$

where the angular brackets denote an ensemble average. We have used the labeling of Takagahara et al. ${ }^{30}$ for the pathways. Mukamel et al. ${ }^{31}$ denote our pathway I as III, and III as I. In our work, $K_{\mathrm{I}}$ defines the coherent pathway whilst the population pathway is given by $K_{\mathrm{II}}+K_{\mathrm{III}}$.

To apply a factorization approximation we average over the bath degrees of freedom for each $G(\omega)$, which we here 
denote by a "bar', notation, such that the $\bar{G}_{\nu \mu}(\omega)$ are complex generalized line-shape functions for the $\nu \leftarrow \mu$ transition, ${ }^{27,32}$

$$
\begin{aligned}
\bar{G}_{\nu \mu}(\omega)= & -i \int_{0}^{\infty} d \tau \exp (i \omega \tau) \\
& \times \exp \left[-i \omega_{\nu \mu} \tau-\frac{1}{2}\left(\gamma_{\nu}+\gamma_{\mu}\right) \tau-g_{\nu \mu}(\tau)\right],
\end{aligned}
$$

with $\gamma_{\nu}^{-1}$ the lifetime of level $\nu, \tau=t_{2}-t_{1}$ and $\omega_{\nu \mu} \equiv E_{\nu}$ $-E_{\mu}$. Such a simplification of the four-time correlation function is based on the assumption of a separation of time scales for the chromophore and bath, such that the average of the product of operators in Eq. (7) factorizes into a product of operator averages (denoted by bars), Eq. (8). This approximation becomes exact in the impact limit (the limit of a short bath correlation time). A more general approach for evaluation of the four-point correlation function is to take the semiclassical limit, then make a weak coupling approximation. ${ }^{27}$ An expression is then obtained which involves the line broadening associated with all pairs of levels.

The middle terms of Eqs. (7) provide the spectral overlap integral (SOI), $J$, which was first noted by Förster, ${ }^{1}$ and essentially ensures energy conservation for the concerted deexcitation-excitation process. The Förster SOI is determined by convolution of the donor emission and acceptor absorption spectra, which gives the probability that an emitted photon (after transit through the bath medium) is absorbed by an acceptor chromophore. In other words, it gauges the extent to which the donor emission and acceptor absorption spectra are "in resonance.' So, for the trivial mechanism, energy conservation between initial and final states is ensured because the photon absorbed by the acceptor is that emitted by the donor, and no ostensibly virtual states are involved. Moreover, the SOI for the trivial mechanism is equivalent to the impact (fast modulation) limit of the population pathway. The dynamics of the fluorescence Stokes' shift have been incorporated in the SOI recently by Mukamel and Rupasov. ${ }^{36}$ Generally, the four-point correlation function is expected to modify the SOI from that derived by Förster. The SOI for the coherent pathway accounts for coherence effects between initial and final states even within the factorization approximation. In the impact limit it reduces to the usual Fermi Golden Rule expression.

\section{B. Coupling matrix elements}

We discuss here the Liouville space matrix elements which promote the energy transfer; beginning with the, essentially, electronic coupling factors. To this end, the coupling is assumed to be independent of nuclear coordinate. The QED matrix elements have a form indicated by

$$
M_{\nu \mu}(\omega) \equiv \sum_{m} V_{m \mu} \bar{G}_{\nu m}(\omega) V_{\nu m}
$$

where we have made a factorization approximation. Owing to the short population time of the virtual intermediate state, this should be a reasonable approximation in the near zone limit. In the usual QED formulation ${ }^{15,18}$ we would have simply $\bar{G}_{\nu m}(\omega)=\left(E_{\nu}-E_{m}\right)^{-1}$; however, by considering Eqs. (3) and (7) we shall here need to evaluate

$$
\begin{aligned}
M_{d a}(\omega)= & \sum_{k, \lambda} \frac{\hbar c k}{2 \epsilon_{0} V} \mu_{d}^{0 r}\left(Q_{d}\right) \mu_{a}^{s 0}\left(Q_{a}\right)\left[-e_{d}^{(\lambda)}(\mathbf{k})\right. \\
& \times \bar{e}_{a}^{(\lambda)}(\mathbf{k}) \exp (i \mathbf{k} \cdot \mathbf{R}) \bar{G}_{d m}(\hbar c k)+e_{a}^{(\lambda)}(\mathbf{k}) \\
& \left.\times \bar{e}_{d}^{(\lambda)}(\mathbf{k}) \exp (-i \mathbf{k} \cdot \mathbf{R}) \bar{G}_{d n}(-\hbar c k)\right] .
\end{aligned}
$$

To evaluate Eq. (10) we first sum over polarizations, then convert the k-sum to an integral. The usual treatment of the coupling may be retrieved by application of the "impact limit," such that $g(\tau)$ is linear in time, $g(\tau)=i \Omega+\hat{\Gamma} \tau$, with $\Omega \equiv \lambda / \sigma$ providing a solvatochromic shift and $\hat{\Gamma} \equiv\left\langle\Delta \omega^{2}\right\rangle / \sigma$ describing the stochastic modulation of the excitation energy leading to line broadening. ${ }^{34}$ This is equivalent to assuming an homogeneous broadening limit, wherein the time scale of bath fluctuations is very fast compared to the rate of the electronic energy transfer process. Hence we put,

$$
\bar{G}_{\nu \mu}(k)=\frac{1}{k-z_{\nu \mu}},
$$

where

$$
c z_{\nu \mu} \equiv \omega_{\nu \mu}+\Omega-i\left(\frac{1}{2} \gamma_{\nu}+\frac{1}{2} \gamma_{\mu}+\hat{\Gamma}_{\nu \mu}\right),
$$

and $k \equiv \omega / c$. Note the equivalence of the formulation involving Eq. (12) with Eqs. (3.1) and (3.2) of Ref. 29. Hence, we obtain

$$
\begin{aligned}
M_{d a}(k)= & \mu_{d}^{0 r}\left(Q_{d}\right) \mu_{a}^{s 0}\left(Q_{a}\right) \int d^{3} \mathbf{k} \frac{k}{2 \epsilon_{0}(2 \pi)^{3}} \\
& \times\left(\delta_{\alpha \beta}-\hat{k}_{\alpha} \hat{k}_{\beta}\right)\left(\frac{e^{i \mathbf{k} \cdot \mathbf{R}}}{z_{d m}-k}-\frac{e^{-i \mathbf{k} \cdot \mathbf{R}}}{z_{d m}+k}\right) .
\end{aligned}
$$

We now put $d^{3} \mathbf{k}=k^{2} d k d \Phi$ and integrate over the solid angle $\Phi$. Keeping in mind that terms odd in $\mathbf{k}$ vanish, we obtain

$$
\begin{aligned}
M_{d a}(k)= & \mu_{d}^{0 r}\left(Q_{d}\right) \mu_{a}^{s 0}\left(Q_{a}\right) \int d k \frac{\exp (i k R)}{4 \pi^{2} i \epsilon_{0} R^{3}} \frac{1}{z_{d m}^{2}-k^{2}} \\
& \times\left[(i k R-1)\left(\delta_{\alpha \beta}-3 \hat{R}_{\alpha} \hat{R}_{\beta}\right)\right. \\
& \left.+k^{2} R^{2}\left(\delta_{\alpha \beta}-\hat{R}_{\alpha} \hat{R}_{\beta}\right)\right] .
\end{aligned}
$$

Integrating over an appropriate semicircular contour into the positive imaginary axis ${ }^{42}$ we finally obtain

$$
\begin{aligned}
M_{d a}(k)= & \mu_{d}^{0 r}\left(Q_{d}\right) \mu_{a}^{s 0}\left(Q_{a}\right) \frac{\exp \left(i z_{d m} R\right)}{4 \pi \epsilon_{0} R^{3}}\left[\left(1-i z_{d m} R\right)\right. \\
& \left.\times\left(\delta_{\alpha \beta}-3 \hat{R}_{\alpha} \hat{R}_{\beta}\right)-\left(z_{d m} R\right)^{2}\left(\delta_{\alpha \beta}-\hat{R}_{\alpha} \hat{R}_{\beta}\right)\right] .
\end{aligned}
$$

In view of the explicit nature of $z_{d m}$, as given by Eq. (12), $\exp \left(i z_{d m} R\right)$ incorporates damping into the rate expression, as investigated also in Refs. 28 and 29. In Ref. 28 [cf. Eq. (4.35)] dielectric effects of the host medium are accounted for using a factor written in the form $y=n \omega / c=\left(n^{\prime}\right.$ 
$\left.+i n^{\prime \prime}\right) \omega / c$. This may be compared to the present work by inspection of Eq. (12) such that $z_{d m}=\left(n^{\prime}+i n^{\prime \prime}\right) \omega / c$, with $n^{\prime}=1+\Omega / \omega$ and $n^{\prime \prime}=-\left(\frac{1}{2} \gamma_{\nu}+\frac{1}{2} \gamma_{\mu}+\hat{\Gamma}_{\nu \mu}\right)$. In vacuum, where $c z_{d m}$ can be exactly identified with $\omega_{d m}$, the exponential in Eq. (15) has a purely imaginary argument and as usual represents an oscillatory phase factor that disappears in the ensuing rate expression. A long-range inverse square dependence on distance is the necessary consequence. However, in dissipative media where $z_{d m}$ acquires complex character, the same exponential also accommodates a damping contribution that tempers the long-range distance dependence.

\section{QED TREATMENT OF THE COUPLING}

The quantum electrodynamical (QED) representation of the interaction between transition moments (which has been discussed previously in the dipole approximation ${ }^{16,18}$ ) will now be developed to higher order. The explicit properties, form and significance of higher multipole contributions to the "near" and "wave"' zone interactions are elucidated.

The interaction Hamiltonian of Eq. (16) couples molecule $N$ to the electromagnetic field. In this way the intermolecular interactions are mediated entirely by the exchange of transverse photons. (It is important to note that the term "transverse"' here refers to the character of the vector field with respect to the propagating vector $\mathbf{k}$. Since the energy transfer process properly accommodates all possible modes for the mediating photon, the resultant coupling displays features both transverse and longitudinal with respect to the intermolecular displacement vector.)

$$
\begin{aligned}
H_{\mathrm{int}}= & \sum_{N}\left\{-\epsilon_{0}^{-1} \mu_{\alpha}(N) d_{\alpha}^{\perp}\left(\mathbf{R}_{N}\right)-\epsilon_{0}^{-1} \theta_{\alpha \beta}(N) \nabla_{\alpha} d_{\beta}^{\perp}\left(\mathbf{R}_{N}\right)\right. \\
& -\epsilon_{0}^{-1} \Omega_{\alpha \beta \gamma}(N) \nabla_{\alpha} \nabla_{\beta} d_{\gamma}^{\perp}\left(\mathbf{R}_{N}\right)-\cdots-m_{\alpha}(N) b_{\alpha}\left(\mathbf{R}_{N}\right) \\
& \left.-m_{\alpha \beta}(N) \nabla_{\alpha} b_{\beta}\left(\mathbf{R}_{N}\right)-\cdots\right\} .
\end{aligned}
$$

Here $\mu_{\alpha}(N), \Theta_{\alpha \beta}(N)$, and $\Omega_{\alpha \beta \gamma}(N)$ are components of the electric dipole $(E 1)$, quadrupole $(E 2)$, and octopole $(E 3)$ transition moment tensor operators, respectively (for molecule $N)$, while $m_{\alpha}(N)$ is the magnetic dipole $(M 1)$ and $m_{\alpha \beta}(N)$ is the magnetic quadrupole (M2) transition moment operator. In Cartesian tensor notation they are defined as

$$
\begin{aligned}
& \mu_{\alpha}(N)=\sum_{i} e_{i}\left(\mathbf{r}_{i}-\mathbf{R}_{N}\right)_{\alpha} \\
& \Theta_{\alpha \beta}(N)=\frac{1}{2 !} \sum_{i} e_{i}\left(\mathbf{r}_{j}-\mathbf{R}_{N}\right)_{\alpha}\left(\mathbf{r}_{i}-\mathbf{R}_{N}\right)_{\beta} \\
& \Omega_{\alpha \beta \gamma}(N)=\frac{1}{3 !} \sum_{i} e_{i}\left(\mathbf{r}_{i}-\mathbf{R}_{N}\right)_{\alpha}\left(\mathbf{r}_{i}-\mathbf{R}_{N}\right)_{\beta}\left(\mathbf{r}_{i}-\mathbf{R}_{N}\right)_{\gamma} \\
& m_{\alpha}(N)=\sum_{i} \frac{e_{i}}{2 m c}\left[\left(\mathbf{r}_{i}-\mathbf{R}_{N}\right) \times \mathbf{p}_{i}\right]_{\alpha} \\
& m_{\alpha \beta}(N)=\frac{1}{2 !} \sum_{i} \frac{e_{i}}{3 m c}\left[\left(\mathbf{r}_{i}-\mathbf{R}_{N}\right) \times \mathbf{p}_{i}\right]_{\alpha}\left[\mathbf{r}_{i}-\mathbf{R}_{N}\right]_{\beta} .
\end{aligned}
$$

Outside the source the transverse displacement vector, $\mathbf{d}^{\perp}\left(\mathbf{R}_{N}\right)$, equals the total electric displacement field at $\mathbf{R}_{N}$ (the position vector of molecule $N$ ), since $\mathbf{d}^{\|}(\mathbf{r})$ is zero for a neutral system.

\section{A. Fully retarded interaction matrix element}

Using second order Rayleigh-Schrödinger perturbation theory (which is equivalent to the impact limit with $\Gamma$ set to zero for clarity) we can write down an expression for the matrix element for the coupling, mediated via the radiation field. ${ }^{16,18}$ Having demonstrated in Sec. II the modulation of the matrix element owing to interactions between the molecule and the host medium, we now focus attention to the explicit form of the multipolar coupling. To this end, and for simplicity, it is appropriate to consider here the vacuum form of the coupling. Hence, proceeding from Sec. II [cf. Eq. (3)] we obtain Eq. (18), which essentially represents the summed interaction between all transition multipoles of the interacting pair,

$$
\begin{aligned}
M_{d a}^{\mathrm{Coul}}= & \sum_{m}\left\langle a\left|H_{\mathrm{int}}\right| m\right\rangle\left\langle m\left|H_{\mathrm{int}}\right| d\right\rangle /\left(E_{d 0}-\hbar c k\right)=\sum_{k, \lambda} \frac{\hbar c k}{2 \epsilon_{0} V}\left\{\left[\mu_{\alpha}^{0 n} \mu_{\beta}^{m 0}+i \hat{k}_{\gamma} k\left(\mu_{\alpha}^{0 n} \Theta_{\beta \gamma}^{m 0}-\Theta_{\alpha \gamma}^{0 n} \mu_{\beta}^{m 0}\right)+\hat{k}_{\gamma} \hat{k}_{\delta} k^{2} \Theta_{\alpha \gamma}^{0 n} \Theta_{\beta \delta}^{m 0}\right.\right. \\
& \left.+\hat{k}_{\gamma} \hat{k}_{\delta} k^{2}\left(\mu_{\alpha}^{0 n} \Omega_{\beta \gamma \delta}^{m 0}+\Omega_{\alpha \gamma \delta}^{0 n} \mu_{\beta}^{m 0}\right)+\cdots\right]\left[e_{\alpha}^{(\lambda)}(\mathbf{k}) \bar{e}_{\beta}^{(\lambda)}(\mathbf{k}) E^{(-)}-e_{\beta}^{(\lambda)}(\mathbf{k}) \bar{e}_{\alpha}^{(\lambda)}(\mathbf{k}) E^{(+)}\right]+\left[\mu_{\alpha}^{0 n} m_{\beta}^{m 0}+i \hat{k}_{\gamma} k\left(\mu_{\alpha}^{0 n} m_{\beta \gamma}^{m 0}\right.\right. \\
& \left.\left.-\Theta_{\alpha \gamma}^{0 n} m_{\beta}^{m 0}\right)\right]\left[e_{\alpha}^{(\lambda)}(\mathbf{k}) b_{\beta}^{(\lambda)}(\mathbf{k}) E^{(-)}-e_{\alpha}^{(\lambda)}(\mathbf{k}) b_{\beta}^{(\lambda)}(\mathbf{k}) E^{(+)}\right]+\left[m_{\alpha}^{0 n} \mu_{\beta}^{m 0}+i \hat{k}_{\gamma} k\left(m_{\alpha}^{0 n} \Theta_{\beta \gamma}^{m 0}-m_{\alpha \gamma}^{0 n} \mu_{\beta}^{m 0}\right)\right]\left[\bar{b}_{\alpha}^{(\lambda)}(\mathbf{k})\right. \\
& \left.\left.\times e_{\beta}^{(\lambda)}(\mathbf{k}) E^{(-)}-b_{\alpha}^{(\lambda)}(\mathbf{k}) \bar{e}_{\beta}^{(\lambda)}(\mathbf{k}) E^{(+)}\right]+m_{\alpha}^{0 n} m_{\beta}^{m 0}\left[b_{\alpha}^{(\lambda)}(\mathbf{k}) \bar{b}_{\beta}^{(\lambda)}(\mathbf{k}) E^{(-)}-b_{\beta}^{(\lambda)}(\mathbf{k}) \bar{b}_{\alpha}^{(\lambda)}(\mathbf{k}) E^{(+)}\right]\right\},
\end{aligned}
$$

where $E^{( \pm)} \equiv \exp (\mp i \mathbf{k} \cdot \mathbf{R}) /\left(E_{n}-E_{0} \pm \hbar c k\right)$. We sum over polarizations using the identities of Eq. (19), where $\epsilon_{\alpha \beta \gamma}$ is the Levi-Civita unit tensor,

$$
\sum_{\lambda} e_{\alpha}^{(\lambda)}(\mathbf{k}) \vec{e}_{\alpha}^{(\lambda)}(\mathbf{k})=\left(\delta_{\alpha \beta}-\hat{k}_{\alpha} \hat{k}_{\beta}\right),
$$

$$
\sum_{\lambda} e_{\alpha}^{(\lambda)}(\mathbf{k}) \vec{b}_{\alpha}^{(\lambda)}(\mathbf{k})=\epsilon_{\alpha \beta \gamma} \hat{k}_{\gamma}
$$$$
\sum_{\lambda} b_{\alpha}^{(\lambda)}(\mathbf{k}) \bar{b}_{\alpha}^{(\lambda)}(\mathbf{k})=\left(\delta_{\alpha \beta}-\hat{k}_{\alpha} \hat{k}_{\beta}\right)
$$ 
We then convert the $\mathbf{k}$-sum to an integral and put $e^{ \pm}$ $\equiv \exp (\mp i \mathbf{k} \cdot \mathbf{R}) /(\kappa \pm k) \quad$ with $\quad \kappa=\left(E_{n}-E_{0}\right) / \hbar c=\left(E_{m}\right.$ $\left.-E_{0}\right) / \hbar c$, so as to obtain

$$
\begin{aligned}
M_{d a}^{\mathrm{Coul}}= & \int d^{3} \mathbf{k}\left(\sum_{P, Q} E_{P}^{0 n} E_{Q}^{m 0}+M_{P}^{0 n} M_{Q}^{m 0}\right) \\
& \times \frac{k}{2 \epsilon_{0}(2 \pi)^{3}}\left(\delta_{\alpha \beta}-\hat{k}_{\alpha} \hat{k}_{\beta}\right)\left[e^{(-)}-e^{(+)}\right] \\
& +\int d^{3} \mathbf{k}\left(\sum_{P, Q} E_{P}^{0 n} M_{Q}^{m 0}+M_{P}^{0 n} E_{Q}^{m 0}\right) \\
& \times \frac{k}{2 \epsilon_{0}(2 \pi)^{3}} \epsilon_{\alpha \beta \gamma} \hat{k}_{\gamma}\left[e^{(-)}-e^{(+)}\right] .
\end{aligned}
$$

Here we have introduced the notation $E_{p}^{0 n}, M_{p}^{0 n}$, etc. where $E$ denotes an electric, and $M$ a magnetic, multipole interaction. The value of $P$ or $Q$ specifies the multipole, so that each contribution from Eq. (18) is specified. The integrations are now performed as in Sec. II, to give the retarded interaction matrix element of Eq. (21), gradients being retrieved from the polarization unit vectors using the relation $\hat{k}_{\gamma}\left[e^{(-)}-e^{(-)}\right]=-i k^{-1} \nabla_{\gamma}\left[e^{(-)}+e^{(-)}\right]$, etc. A detailed derivation of Eq. (21) has been presented previously, ${ }^{40}$

$$
\begin{aligned}
M_{d a}^{\mathrm{Coul}}= & \left(4 \pi \epsilon_{0}\right)^{-1}\left[\mu_{\alpha}^{0 n} \mu_{\beta}^{m 0}+\left(\mu_{\alpha}^{0 n} \Theta_{\beta \gamma}^{m 0}-\Theta_{\alpha \gamma}^{0 n} \mu_{\beta}^{m 0}\right) \nabla_{\gamma}\right. \\
& +\Theta_{\alpha \gamma}^{0 n} \Theta_{\beta \delta}^{m 0} \nabla_{\gamma} \nabla_{\delta}+\left(\mu_{\alpha}^{0 n} \Omega_{\beta \gamma \delta}^{m 0}+\Omega_{\alpha \gamma \delta}^{0 n} \mu_{\beta}^{m 0}\right) \nabla_{\gamma} \nabla_{\delta} \\
& \left.+m_{\alpha}^{0 n} m_{\beta}^{m 0}\right]\left(\nabla^{2} \delta_{\alpha \beta}-\nabla_{\alpha} \nabla_{\beta}\right) R^{-1} \exp (i \kappa R) \\
& +\left(4 \pi \epsilon_{0}\right)^{-1}\left[i \mu_{\alpha}^{0 n} m_{\beta}^{m 0}+i m_{\alpha}^{0 n} \mu_{\beta}^{m 0}+\left(\mu_{\alpha}^{0 n} m_{\beta \delta}^{m 0}\right.\right. \\
& \left.\left.+m_{\alpha \delta}^{0 n} \mu_{\beta}^{m 0}\right) \nabla_{\delta}+\left(m_{\alpha}^{0 n} \Theta_{\beta \delta}^{m 0}-\Theta_{\alpha \delta}^{0 n} m_{\beta}^{m 0}\right) \nabla_{\delta}\right] \\
& \times \epsilon_{\alpha \beta \gamma} \nabla_{\gamma} R^{-1} \kappa \exp (i \kappa R) .
\end{aligned}
$$

We now rewrite Eq. (21) in the form

$$
\begin{aligned}
M_{d a}^{\mathrm{Coul}}= & {\left[\left(\mu_{\alpha}^{0 n} \mu_{\beta}^{m 0}+m_{\alpha}^{0 n} m_{\beta}^{m 0}\right) V_{\alpha \beta}+\left(\mu_{\alpha}^{0 n} \Theta_{\beta \gamma}^{m 0}\right.\right.} \\
& \left.-\Theta_{\beta \gamma}^{0 n} \mu_{\alpha}^{m 0}\right) V_{\alpha \beta \gamma}+\left(\Theta_{\alpha \gamma}^{0 n} \Theta_{\beta \delta}^{m 0}+\mu_{\alpha}^{0 n} \Omega_{\beta \gamma \delta}^{m 0}\right. \\
& \left.+\Omega_{\beta \gamma \delta}^{0 n} \mu_{\alpha}^{m 0}\right) V_{\alpha \beta \gamma \delta}+\cdots+\operatorname{Im}\left(\mu_{\alpha}^{0 n} m_{\beta}^{m 0}\right. \\
& \left.+m_{\beta}^{0 n} \mu_{\alpha}^{m 0}\right) V_{\alpha \beta}^{\prime}+\operatorname{Im}\left(m_{\alpha}^{0 n} \Theta_{\beta \delta}^{m 0}-\Theta_{\alpha \delta}^{0 n} m_{\beta}^{m 0}\right) V_{\alpha \beta \gamma}^{\prime} \\
& \left.+\operatorname{Im}\left(\mu_{\alpha}^{0 n} m_{\beta \gamma}^{m 0}+m_{\beta \gamma}^{0 n} \mu_{\alpha}^{m 0}\right) V_{\alpha \beta \gamma}^{\prime}+\cdots\right],
\end{aligned}
$$

where it may be noted that the magnetic transition moment tensors are purely imaginary, that is, $-i m_{\alpha}=\operatorname{Im}\left(m_{\alpha}\right)$, etc. The $V_{j}$ are the transition moment coupling tensors discussed below. Note that those associated with $E-E$ or $M-M$ interactions differ from those for $E-M$ coupling (highlighted by the primes), essentially owing to their different time-reversal signature.

The structure of the $E-E$ and $M-M$ coupling tensor is given by $V_{i_{1} i_{2} \cdots i_{n}}=\nabla_{i_{1}} \cdots \nabla_{i_{n-2}}\left(\nabla^{2} \delta_{i_{n-1} i_{n}}-\nabla_{i_{n-1}} \nabla_{i_{n}}\right)$ $\times\left(e^{i \kappa R} / R\right)$, which runs as $R^{-(n+1)}$, where $\left(e^{i \kappa R} / R\right)$ can be identified with $i \kappa h_{0}^{(1)}(\kappa R)$, a spherical Bessel function of the third kind. ${ }^{44}$ Similarly, coupling tensors associated with the $E-M$ cross terms have the general form $V_{i_{1} i_{2} \cdots i_{n}}^{\prime}$ $=\left(4 \pi \epsilon_{0}\right)^{-1}\left[\nabla_{i_{1}} \nabla_{i_{2}} \cdots \nabla_{i_{n-3}}\left(\epsilon_{i_{n-2} i_{n-1} i_{n}} \nabla_{i_{n}}\right)\left(\kappa e^{i \kappa R} / R\right)\right]$.
The spherical Bessel function is defined in terms of the Hankel function of order integer plus half as $h_{n}^{(1)}(z)$ $=(\pi / 2 z)^{1 / 2} H_{n+(1 / 2)}^{(1)}(z)$, and is given by

$$
h_{n}^{(1)}(z)=i^{-n-1} \frac{e^{i z}}{z} \sum_{k=0}^{n}(-1)^{k} \frac{(n+k) !}{k !(n-k) !} \frac{1}{(2 i z)^{k}} \text {. }
$$

We can now evaluate the gradients of Eq. (21) by use of the identities collected in the following equations:

$$
\begin{aligned}
& \nabla_{\alpha} R=\hat{R}_{\alpha}, \\
& \nabla_{\alpha} \hat{R}_{\beta}=\frac{1}{R}\left(\delta_{\alpha \beta}-\hat{R}_{\alpha} \hat{R}_{\beta}\right), \\
& \nabla_{\alpha} \exp (i \kappa R)=i \kappa \hat{R}_{\alpha} \exp (i \kappa R), \\
& \nabla_{\alpha} h_{n}^{(1)}(\kappa R)=\left[\frac{n}{R} h_{n}^{(1)}(\kappa R)-\kappa h_{n+1}^{(1)}(\kappa R)\right] \hat{R}_{\alpha} .
\end{aligned}
$$

Finally, we obtain expressions for the coupling tensors given in Eqs. (25) and (26). It is evident that the use of the spherical Bessel function formulation provides a clear, compact representation of the retarded coupling interaction and its distance-dependence,

$$
\begin{aligned}
& V_{\alpha \beta}(\kappa, \mathbf{R})=\frac{-i \kappa^{3}}{4 \pi \epsilon_{0}}\left[\delta_{\alpha \beta} h_{0}^{(1)}(\kappa R)-\frac{1}{\kappa R} \delta_{\alpha \beta} h_{1}^{(1)}(\kappa R)\right. \\
& \left.+\hat{R}_{\alpha} \hat{R}_{\beta} h_{2}^{(1)}(\kappa R)\right] \\
& V_{\alpha \beta \gamma}(\kappa, \mathbf{R})=\frac{i \kappa^{4}}{4 \pi \epsilon_{0}}\left[\delta_{\alpha \beta} \hat{R}_{\gamma} h_{1}^{(1)}(\kappa R)-\frac{1}{\kappa R}\right. \\
& \times\left(\delta_{\alpha \beta} \hat{R}_{\gamma}+\delta_{\alpha \gamma} \hat{R}_{\beta}+\delta_{\beta \gamma} \hat{R}_{\alpha}\right) h_{2}^{(1)}(\kappa R) \\
& \left.+\hat{R}_{\alpha} \hat{R}_{\beta} \hat{R}_{\gamma} h_{3}^{(1)}(\kappa R)\right] \text {, } \\
& V_{\alpha \beta \gamma \delta}(\kappa, \mathbf{R})=\frac{i \kappa^{5}}{4 \pi \epsilon_{0}}\left[\delta_{\alpha \beta} \delta_{\gamma \delta} h_{1}^{(1)}(\kappa R)-\frac{1}{\kappa^{2} R^{2}}\left(\delta_{\alpha \beta} \delta_{\gamma \delta}\right.\right. \\
& \left.+\delta_{\alpha \gamma} \delta_{\beta \delta}+\delta_{\alpha \delta} \delta_{\beta \gamma}\right) h_{2}^{(1)}(\kappa R) \\
& -\delta_{\alpha \beta} \hat{R}_{\gamma} \hat{R}_{\delta} h_{2}^{(1)}(\kappa R)+\frac{1}{\kappa R}\left(\delta_{\alpha \beta} \hat{R}_{\gamma} \hat{R}_{\delta}\right. \\
& +\delta_{\alpha \gamma} \hat{R}_{\beta} \hat{R}_{\delta}+\delta_{\alpha \delta} \hat{R}_{\beta} \hat{R}_{\gamma}+\delta_{\beta \gamma} \hat{R}_{\alpha} \hat{R}_{\delta} \\
& \left.+\delta_{\beta \delta} \hat{R}_{\alpha} \hat{R}_{\gamma}+\delta_{\gamma \delta} \hat{R}_{\alpha} \hat{R}_{\beta}\right) h_{3}^{(1)}(\kappa R) \\
& \left.-\hat{R}_{\alpha} \hat{R}_{\beta} \hat{R}_{\gamma} \hat{R}_{\delta} h_{4}^{(1)}(\kappa R)\right] \\
& V_{\alpha \beta}^{\prime}(\kappa, \mathbf{R})=\frac{i \kappa^{3}}{4 \pi \epsilon_{0}} \epsilon_{\alpha \beta \gamma} \hat{R}_{\gamma} h_{1}^{(1)}(\kappa R), \\
& V_{\alpha \beta \gamma}^{\prime}(\kappa, \mathbf{R})=\frac{i \kappa^{4}}{4 \pi \epsilon_{0}}\left[\frac{1}{\kappa R} \epsilon_{\alpha \beta \delta} \delta_{\gamma \delta} h_{1}^{(1)}(\kappa R)\right. \\
& \left.-\epsilon_{\alpha \beta \delta} \hat{R}_{\gamma} \hat{R}_{\delta} h_{2}^{(1)}(\kappa R)\right] \text {. }
\end{aligned}
$$


Equations (25)-(26) proves a more amenable basis for derivation of the higher order coupling than the algebraically equivalent linear combination of spherical Bessel functions employed in previous work. ${ }^{45}$ These higher order coupling tensors may be used to obtain improved estimates of the distance and orientation dependence of energy transfer, as discussed in Sec. IV.

\section{B. Irreducible tensor formulation}

It is instructive to consider the coupling between donor and acceptor transition multipoles in terms of irreducible tensors. Consider the coupling between a donor electric or magnetic transition multipole moment, $E p$ or $M p(p=1$ denoting dipole, $p=2$ quadrupole, etc.) written for generality as $D_{i 1 \ldots i p}$, and an acceptor multipole $E q$ or $M q$, written as $A_{i 1 \ldots i q}$. Each multipole as given explicitly by Eqs. (17) is in general expressible as a sum of irreducible tensors, $D_{i_{1} \ldots i_{p}}^{(j)}$ and $A_{i_{1} \ldots i q}^{(j)}$, characterized by integer weights $j=0 \cdots p(q)$. These are tensors which have the transformation properties of angular momenta $j$ under the symmetry operations of the full rotation-inversion group $\mathrm{SO}(3)$. When a donor multipole of rank $p$ couples with an acceptor multipole of rank $q$, the coupling tensor has rank $p+q$ and in principle accommodates weights running from $|p-q|$ up to $p+q$.

Not all weights $j=0 \cdots p(q)$ are manifest however. First, because each multipole is fully symmetric with respect to interchange of any of its indices, those weights for which $(p-j)$ or $(q-j)$ are odd, vanish. ${ }^{46}$ Second, the $j=0$ contributions, which need therefore only be considered for even $p(q)$, necessarily vanish for a quite different reason. This is because on contraction with, for example, $\nabla_{i_{1}} \cdots \nabla_{i_{p-1}} d_{i_{p}}^{\perp}$, the even rank weight 0 tensors, constructed from a product of Kronecker deltas, lead to a factor $\nabla \cdot d^{\perp}$. This divergence term is zero, the electric displacement field in the multipolar gauge being explicitly transverse. The same argument applies for the magnetic multipoles, as the magnetic field of electromagnetic radiation is intrinsically divergence-free. Thus the weights run from (at least) 1 up to $p(q)$ if the rank is odd, and from (at least) 2 up to $p(q)$ if the rank is even. Notice that weights different from the rank arise for $p(q)$ $>2$, so that the higher multipoles are not natural tensors. This is a feature that has only recently been signaled in connection with electric octopole coupling, ${ }^{47}$ where the weight 1 part of the rank 3 tensor has often in the past escaped notice. ${ }^{48}$ For example, $V_{\alpha \beta \gamma}$ carries only weights 1 and 3 , as can be demonstrated by explicit decomposition ${ }^{49-51}$ - the result is as follows:

$$
V_{\alpha \beta \gamma}(\kappa, \mathbf{R})=V_{\alpha \beta \gamma}^{(1)}(\kappa, \mathbf{R})+V_{\alpha \beta \gamma}^{(3)}(\kappa, \mathbf{R})
$$

with

$$
\begin{aligned}
V_{\alpha \beta \gamma}^{(1)}(\kappa, \mathbf{R})= & \frac{i \kappa^{4}}{4 \pi \epsilon_{0}}\left[h_{1}^{(1)}(\kappa R)-\frac{1}{\kappa R} h_{2}^{(1)}(\kappa R)\right. \\
& \left.+\frac{1}{5} h_{3}^{(1)}(\kappa R)\right] \delta_{\alpha \beta} \hat{R}_{\gamma}+\left[\frac{-1}{\kappa R} h_{2}^{(1)}(\kappa R)\right.
\end{aligned}
$$

$$
\begin{aligned}
& \left.+\frac{1}{5} h_{3}^{(1)}(\kappa R)\right]\left(\delta_{\alpha \gamma} \hat{R}_{\beta}+\delta_{\beta \gamma} \hat{R}_{\alpha}\right), \\
V_{\alpha \beta \gamma}^{(3)}(\kappa, \mathbf{R})= & \frac{i \kappa^{4}}{4 \pi \epsilon_{0}} h_{3}^{(1)}(\kappa R)\left[\hat{R}_{\alpha} \hat{R}_{\beta} \hat{R}_{\gamma}-\frac{1}{5}\left(\delta_{\alpha \beta} \hat{R}_{\gamma}\right.\right. \\
& \left.\left.+\delta_{\alpha \gamma} \hat{R}_{\beta}+\delta_{\beta \gamma} \hat{R}_{\alpha}\right)\right] .
\end{aligned}
$$

The evident absence of weight 2 contributions reflects the paired effect of the $\alpha, \beta$ symmetry ensuing from the index symmetry of the $V_{\alpha \beta}$ of Eq. (25a), from which the $V_{\alpha \beta \gamma}$ is derived, and the quite separate $\beta, \gamma$ symmetry arising because the coupling tensor contracts with the index symmetric quadrupole transition moment in Eq. (22).

The irreducible tensor decomposition of the coupling tensor $V_{n}$ shows that for even $n$ the $R$-dependence of the weight 0 part which then arises always runs as $h_{0}^{(1)}(\kappa R)$; a spherical Bessel function of the third kind. This can be seen from the fact that the weight 0 contribution can be obtained by tracing the product of nabla operators, necessarily leading to a product of $(n / 2)$ Laplacians, $\left(\nabla^{2}\right)^{n / 2}$. Since the Laplacian can here be written as $R^{-1}\left(\partial^{2} / \partial R^{2}\right) R$, its successive operation on $\left(e^{i \kappa R} / R\right)$ simply introduces the multiplicative factor $(i \kappa)^{n}$ into the same function.

For electric-electric or magnetic-magnetic interactions, each contribution to the matrix element for the coupling is clearly expressible in the form

$$
D_{p} A_{q} V_{p+q}=\sum_{j} \sum_{j^{\prime}} \sum_{j^{\prime \prime}} D_{p}^{(j)} A_{q}^{\left(j^{\prime}\right)} V_{p+q}^{j^{\prime \prime}},
$$

using the single subscript on each tensor to represent its full set of indices. The product $D_{p}^{(j)} A_{q}^{\left(j^{\prime}\right)}$, for the reasons outlined above, is a tensor of rank $p+q$ which contains even weights $j^{\prime \prime \prime}$ running from 0 up to $p+q$ if the latter sum is even, or odd weights $j^{\prime \prime \prime}$ from 1 up to $p+q$ if the sum is odd. In coupling this resultant tensor with $V_{p+q}$ only the products with $j^{\prime \prime \prime}=j^{\prime \prime}$ can contribute since the result is necessarily a scalar. This has a significant bearing on the form of result that ensues on rotational averaging, as will be shown in Sec. III C.

Hence, not only additional physical insight but also a significant degree of simplification of Eq. (22) is achieved by representing the donor and acceptor transition multipole moments as sums of their nonvanishing irreducible components [i.e., weights $1, \ldots, p(q)$ for a multipole of odd rank or weights $2, \ldots, p(q)$ for a multipole of even rank], then considering the nonvanishing terms in the multipole product tensors, $D_{p}^{(j)} A_{q}^{\left(j^{\prime}\right)}$, and the coupling tensors, $V_{p+q}$, as discussed above.

\section{Rotational averages}

In systems that exhibit resonance energy transfer between structurally uncorrelated centres, the theory as developed above stands in need of further refinement before its results can be related to experiment. In such systems, including microscopically disordered solids, glasses and fluids, it is 
necessary to relate theory to measurement by performing rotational averages to account for the distribution of donor and acceptor orientations. For simplicity we shall proceed on the assumption of an isotropic orientational distribution, in which the donor and acceptor are orientationally uncorrelated, though partially ordered systems can properly be addressed by application of a related procedure also based on irreducible tensors. ${ }^{52,53}$

Through application of a procedure described in detail elsewhere $^{54}$ the implementation of such an average (denoted below by angular brackets), on the rate equation whose root is Eq. (29), gives a result expressible as

$$
\begin{aligned}
\left\langle\left|D_{p} A_{q} V_{p+q}\right|^{2}\right\rangle= & \sum_{j} \sum_{j^{\prime}} \sum_{j^{\prime \prime}}\left\langle D_{p}^{(j)} \bar{D}_{p}^{(j)}\right\rangle\left\langle A_{q}^{\left(j^{\prime}\right)} \bar{A}_{q}^{\left(j^{\prime}\right)}\right\rangle \\
& \times\left\langle V_{p+q}^{j^{\prime \prime}} \bar{V}_{p+q}^{j^{\prime \prime}}\right\rangle,
\end{aligned}
$$

where $j, j^{\prime}$, and $j^{\prime \prime}$ satisfy the rules of angular momentum coupling as outlined in the previous section. For our purposes the key feature is $\left\langle V_{p+q}^{j^{\prime \prime}} \bar{V}_{p+q}^{j^{\prime \prime}}\right\rangle$, which carries identifiable contributions.

We take $E 1-E 1$ coupling as an example. The form of the spherical Bessel functions which comprise the coupling tensor $V_{\alpha \beta}(\kappa, \mathbf{R})$ given in Eq. (25) dictates the form of the rate expression. The second rank coupling tensor can be reduced into two nonvanishing irreducible components such that,

$$
V_{\alpha \beta}(\kappa, \mathbf{R})=V_{\alpha \beta}^{(0)}(\kappa, \mathbf{R})+V_{\alpha \beta}^{(2)}(\kappa, \mathbf{R})
$$

with

$$
\begin{aligned}
V_{\alpha \beta}^{(0)}(\kappa, \mathbf{R})= & \frac{-i \kappa^{3}}{4 \pi \epsilon_{0}}\left[h_{0}^{(1)}(\kappa R)-\frac{1}{\kappa R} h_{1}^{(1)}(\kappa R)\right. \\
& \left.+\frac{1}{3} h_{2}^{(1)}(\kappa R)\right] \delta_{\alpha \beta}, \\
V_{\alpha \beta}^{(2)}(\kappa, \mathbf{R})= & \frac{-i \kappa^{3}}{4 \pi \epsilon_{0}} h_{2}^{(1)}(\kappa R)\left(\hat{R}_{\alpha} \hat{R}_{\beta}-\frac{1}{3} \delta_{\alpha \beta}\right) .
\end{aligned}
$$

The form of the spherical Bessel functions dictates that the former contribution is only significant in the wave zone. In the fully averaged rate equations the following factors arise:

$$
\begin{aligned}
V_{\alpha \beta}^{(0)}(\kappa, \mathbf{R}) \bar{V}_{\alpha \beta}^{(0)}(\kappa, \mathbf{R})= & \frac{\omega^{4}}{12 \pi^{2} \epsilon_{0}^{2} c^{4} R^{2}}, \\
V_{\alpha \beta}^{(2)}(\kappa, \mathbf{R}) \bar{V}_{\alpha \beta}^{(2)}(\kappa, \mathbf{R})= & \frac{\omega^{4}}{24 \pi^{2} \epsilon_{0}^{2} c^{4}} \\
& \times\left(\frac{1}{R^{2}}+\frac{3 c^{2}}{\omega^{2} R^{4}}+\frac{9 c^{4}}{\omega^{4} R^{6}}\right) .
\end{aligned}
$$

The rotational average given by the final factor in Eq. (31) is then expressible as

$$
\begin{aligned}
A(\kappa, \mathbf{R}) & =V_{\alpha \beta}^{(0)}(\kappa, \mathbf{R}) \bar{V}_{\alpha \beta}^{(0)}(\kappa, \mathbf{R})+V_{\alpha \beta}^{(2)}(\kappa, \mathbf{R}) \bar{V}_{\alpha \beta}^{(2)}(\kappa, \mathbf{R}) \\
& =2\left(4 \pi \epsilon_{0} R^{3}\right)^{-2}\left[3+(\kappa R)^{2}+(\kappa R)^{4}\right]
\end{aligned}
$$

which is the familiar excitation transfer function for $E 1-E 1$ coupling. ${ }^{18}$ The wave-zone dependence of the rate is thus established as $R^{-2}$, a result which necessarily holds for all multipolar couplings. This ensures radiative energy conservation - the net energy flux through a shell at a wavezone distance $R \gg \kappa^{-1}$ from the donor must be imperceptibly dependent on the distance from the source.

\section{DISCUSSION}

The QED framework affords a general and lucid formulation for examining the mechanism of energy transfer. ${ }^{13-21,55}$ Host dynamics have been incorporated into the QED model in Sec. II of the present work, properly allowing us to identify the partitioning between the near and far zone mechanisms for transfer of energy between chromophores dispersed in condensed phase (liquid or solid) host media. It has been shown that the form of the phase factor associated with the coupling acquires a complex character in dissipative media, tending to moderate the long-range distance dependence (typically donor-acceptor separations of greater than $100 \mathrm{~nm}$ ). A previous study ${ }^{28}$ has associated this exponential decay factor with a quenching of bath polaritons involved in mediating the EET. Such a subsystem is necessarily dissipative because of the dense excitation spectrum of the medium.

Coherent and "population" Liouville space pathways have been investigated in Sec. II B. It was shown that, in the fast modulation (homogeneous broadening) limit, spectral overlap integrals representative of each pathway may be identified. They may be compared to the Förster expression, which essentially represents the limit of a "trivial" (photon emission-absorption) mechanism. At close separations (typically less than $100 \mathrm{~nm}$ ), the near zone, the exponential phase and damping factors identified in Eq. (15) vanish, and the influence of the host medium enters only via the spectral overlap integral. Screening contributions and local field effects owing to the dielectric properties of the host medium may be considered also, as was shown recently. ${ }^{28,56}$ For small interchromophore separations (e.g., less than about 20-30 A), typical of model bichromophores, aromatic polymers, or photosynthetic light harvesting complexes, the host medium refractive index does not feature in the rate equation, although the rate expression may be modified my microscopic solvent effects. ${ }^{57}$ When the donor-acceptor separation greatly exceeds the host molecule "radius", (i.e., a large number of host/solvent molecules may be accommodated between donor and acceptor chromophores), then the refractive index factor $\left(l / n^{4}\right.$ in the Förster expression) should be included. ${ }^{28,57}$

A general QED treatment of higher multipole contributions to the coupling between transition moments has been presented in Sec. III, whence interactions involving electric dipole, quadrupole, and octopole as well as magnetic dipole and quadrupole interactions were examined. The utility and need for the establishment of such a physical picture in the QED formalism has been highlighted by a recent study of the coupling between the carotenoid $S_{1}$ state and chlorophylls. ${ }^{38}$ 
A new formulation has been presented wherein expressions for the multipolar coupling tensors are constructed in terms of spherical Bessel functions, providing a clear, compact representation of the retarded coupling interaction and its distance-dependence [cf. Eqs. (22), (25), and (26)]. It has been shown that, starting from Eqs. (25a) and (26a), higher coupling tensors are obtained easily by successive application of Eq. (24d).

The most common regime for which higher multipole interactions may require consideration in a study of electronic energy transfer is the near zone (i.e., at close separations, where $\kappa R \ll 1)$. In this limit, the interaction tensors of Eqs. (25) and (26) reduce to those of Eqs. (35) and (36) below:

$$
\begin{aligned}
V_{\alpha \beta} \approx & \frac{1}{4 \pi \epsilon_{0}}\left(\delta_{\alpha \beta}-3 \hat{R}_{\alpha} \hat{R}_{\beta}\right) / R^{3} \\
V_{\alpha \beta \gamma} \approx & \frac{1}{4 \pi \epsilon_{0}}\left[15 \hat{R}_{\alpha} \hat{R}_{\beta} \hat{R}_{\gamma}-3\left(\delta_{\alpha \beta} \hat{R}_{\gamma}+\delta_{\alpha \gamma} \hat{R}_{\beta}\right.\right. \\
& \left.\left.+\delta_{\beta \gamma} \hat{R}_{\alpha}\right)\right] / R^{4} \\
V_{\alpha \beta \gamma \delta} \approx & \frac{1}{4 \pi \epsilon_{0}}\left[-105 \hat{R}_{\alpha} \hat{R}_{\beta} \hat{R}_{\gamma} \hat{R}_{\delta}-3\left(\delta_{\alpha \beta} \delta_{\gamma \delta}+\delta_{\alpha \gamma} \delta_{\beta \delta}\right.\right. \\
& \left.+\delta_{\alpha \delta} \delta_{\beta \gamma}\right)+15\left(\delta_{\alpha \beta} \hat{R}_{\gamma} \hat{R}_{\delta}+\delta_{\alpha \gamma} \hat{R}_{\beta} \hat{R}_{\delta}\right. \\
& +\delta_{\alpha \delta} \hat{R}_{\beta} \hat{R}_{\gamma}+\delta_{\beta \gamma} \hat{R}_{\alpha} \hat{R}_{\delta}+\delta_{\beta \delta} \hat{R}_{\alpha} \hat{R}_{\gamma} \\
& \left.\left.+\delta_{\gamma \delta} \hat{R}_{\alpha} \hat{R}_{\beta}\right)\right] / R^{5}, \\
V_{\alpha \beta}^{\prime} \approx & \frac{1}{4 \pi \epsilon_{0}} \epsilon_{\alpha \beta \gamma} \hat{R}_{\gamma} \kappa / R^{2}, \\
V_{\alpha \beta \gamma}^{\prime} \approx & \frac{1}{4 \pi \epsilon_{0}} \epsilon_{\alpha \beta \delta}\left(\delta_{\gamma \delta}-3 \hat{R}_{\gamma} \hat{R}_{\delta}\right) \kappa / R^{3} .
\end{aligned}
$$

In the spherical Bessel function representation, these equations are found by taking the limiting values of the $h_{n}^{(1)}(\kappa R)$ for small arguments. The near zone interaction has a form reminiscent of the interaction between two static dipoles, although the irreducible tensor formulation of the coupling discussed in Sec. III B signals features that have often in the past escaped notice. At very large donor-acceptor separations (i.e., in the wave zone, with $\kappa R \gg 1$ ) all multipolar couplings assume a $\kappa^{2} / R$ distance dependence, consistent with Poynting vector calculations.

Orientation factors for the multipolar coupling are obtained by making the substitutions $\delta_{\alpha \beta} \rightarrow \alpha \cdot \beta$ and $\hat{R}_{\alpha} \rightarrow \alpha$ $\cdot \hat{\mathbf{R}}$, where $\alpha$ denotes a unit vector in the direction of tensor component $\alpha$ of the transition moment and $\hat{\mathbf{R}}$ specifies the direction of the center-to-center vector. Directions of the transition moment tensor components are depicted in Fig. 3. When the dipole transition moments of donor and acceptor are normal to $\hat{\mathbf{R}}$, we obtain $\cos \varphi, 0$ and $-(3 / 2) \cos \varphi$ for the orientation factors for Eqs. (35a), (35b), and (35c), respectively, where $\varphi$ is the angle between these dipole transition

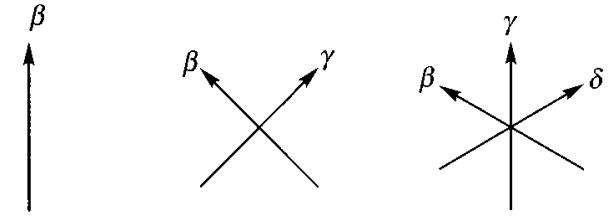

FIG. 3. Illustration of the directions of multipolar transition moment tensor components; dipole, quadrupole, and octopole (left to right).

moments. However, all multipole transition moments contribute to the coupling whenever $\theta_{a}$ and $\theta_{b}$ (see Ref. 6) are different from $90^{\circ}$.

The relative weight of the transition multipole moments may be estimated by recourse to the approximate relations, $|m| c \approx|\mu| \alpha,|\theta| \approx|\mu| l,|\Omega| \approx|\mu| l^{2}$, etc., with $\alpha=1 / 137$ and $l$ the length of the transition dipole. From such considerations one would expect that at the interchromophore separation $R=l$, all $E p-E q$ interactions should contribute equally, which is congruent with the dipole approximation being considered valid for $R \gg l$. This was investigated recently for various model dimer separations. ${ }^{6,37}$

Examination of the form for the coupling tensors for the electric-magnetic cross terms $E p-M q, V^{\prime}$, shows that the interaction depends always upon $\kappa$, even in the near zone. Thus these constitute examples of "entirely retarded" couplings. ${ }^{58}$ Similarly, contributions to the coupling outside the wave zone also involve an explicit $\kappa$-dependence. Consideration of Sec. II C (which leads to the substitution of $z_{d m}$-type terms for $\kappa$ ) suggests the damping owing to a dissipative host medium should be enhanced for these interactions. In general, the interactions involving magnetic multipole transition moments will be significantly smaller than those involving electric multipole moments owing to this $\kappa$-dependence of the coupling, which is typically equivalent to a factor of $1 /(5000 \AA)$.

Using the theory developed in the present work, a detailed study has been undertaken of the convergence of the multipolar expansion of the near zone coupling between various chromophores, including bacteriochlorophyll $a$, as a function of separation and orientation. ${ }^{60}$ It was found that corrections to the dipole approximation from higher multipole couplings are significant to fairly high order at separations typical of those in biological light-harvesting aggregates. The reason for this is simply that the transition moments do not behave as ideal dipoles. A feel for the problem is obtained by considering the axial components of the dipole, quadrupole, and octopole transition moments calculated for the bacteriochlorophyll $a$ Q $Q_{y}$ transition; $\mu_{y}$ $\approx 14 \mathrm{D}, \Theta_{y y} \approx-9 \mathrm{D} \AA$, and $\Omega_{y y y} \approx 225 \mathrm{D} \AA$. $^{2,60}$ It is clear that interactions involving the quadrupole transition moment will be relatively small compared to those involving the dipole and octopole (as expected for an allowed transition ${ }^{5,6}$ ). By using these transition multipole moments together with Eqs. (22) and (35) we can easily determine the ratio of, for example, the dipole-dipole coupling to the dipole-octopole coupling (i.e., $M^{\mathrm{do}} / M^{\mathrm{dd}}$ ). By using the orientation depicted 


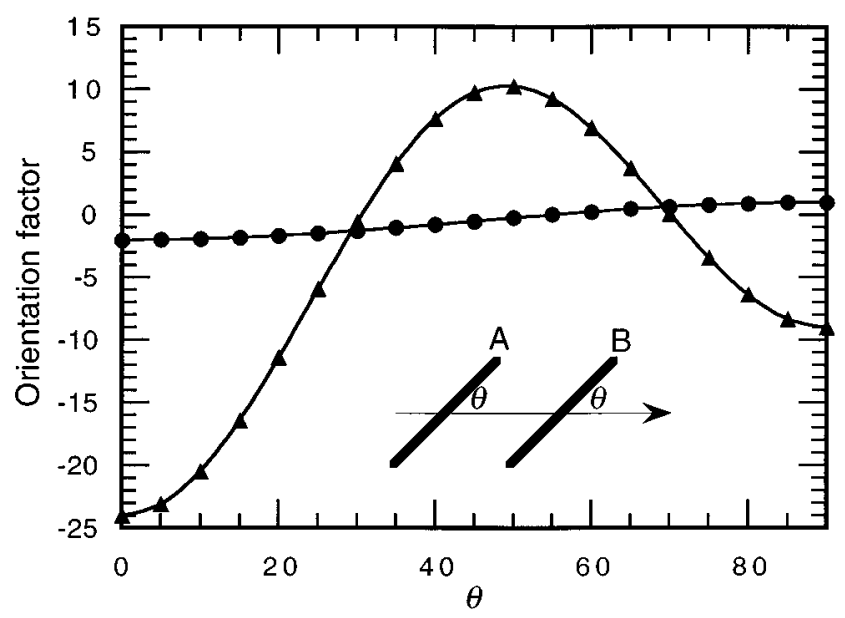

FIG. 4. Comparison of orientation factors for the dipole-dipole and dipoleoctopole couplings between bacteriochlorophyll $a Q_{y}$ transitions, calculated using Eqs. (35), for the orientation depicted in the inset.

in Fig. 4, with $\theta=65^{\circ}$ (such that the orientation factor for dipole-dipole coupling is 0.46 and that for dipole-octopole coupling is 3.73) we obtain $M^{\mathrm{do}} / M^{\mathrm{dd}}$ as a function of separation (Fig. 5). This shows that, for bacteriochlorophyll $a$, the higher multipole couplings are very significant and the expansion should be taken to fairly high order to calculate couplings with reasonable accuracy. This is not so surprising given the size of these chromophores. A more detailed analysis is provided in Ref. 60.

\section{CONCLUSIONS}

(i) The interplay between electronic coupling, spectral linewidth and rate of electronic energy transfer between chromophores was examined in the context of a quantum electrodynamical (QED) model, allowing us to identify the partitioning between the near and far zone mechanisms for transfer of energy between chromophores dispersed in condensed phase (liquid or solid) host media. The long-range distancedependence of the coupling was shown to be modified by a phase factor, through which damping effect were manifest.

(ii) A general QED treatment of higher multipole contributions to the coupling between transition moments was derived. A new formulation was presented wherein expressions for the multipolar coupling tensors were obtained in terms of spherical Bessel functions, providing a clear, compact representation of the retarded coupling interaction and its distancedependence for multipolar couplings of arbitrary order. The irreducible tensor formulation of the coupling was discussed, highlighting features concerning the exact form of the orientation factors that have often in the past escaped notice, and the detailed method of implementing a rotational averaging of the resultant interaction tensors was demonstrated. The coupling between bacteriochlorophyll $a$ chromophores

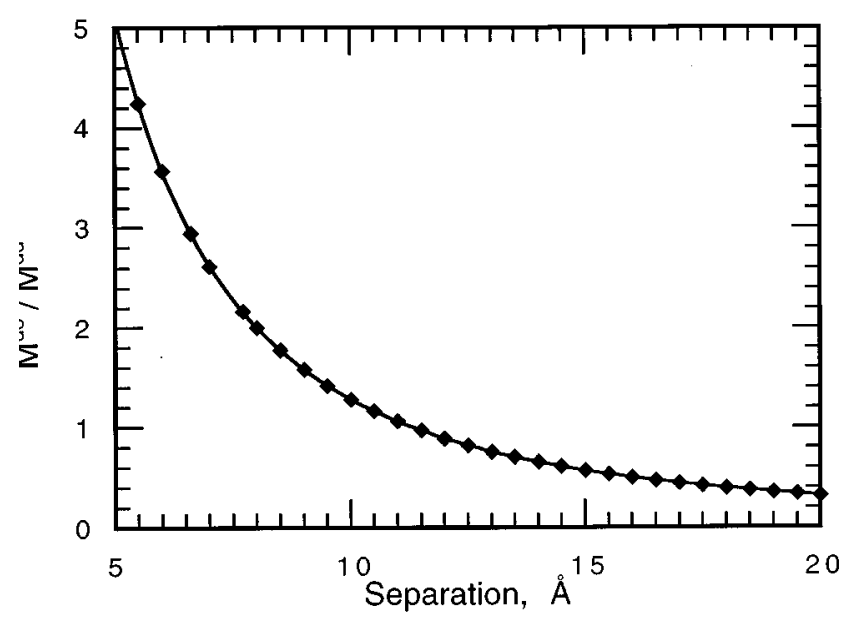

FIG. 5. Distance-dependence of the ratio of the dipole-octopole to dipoledipole couplings between bacteriochlorophyll $a Q_{y}$ transitions, calculated using Eqs. (22) and (35), for the orientation depicted in Fig. 4 with $\theta$ $=65^{\circ}$.

was examined as an example; illustrating the real significance of higher multipolar couplings for separations less than $20 \AA$.

\section{ACKNOWLEDGMENTS}

The success of this work owes much to the following individuals with whom we have had highly profitable discussions: Dr. T. Thirunamachandran, Dr. A. Bittner, Dr. P. Allcock, and Professor G. R. Fleming. G.D.S. gratefully acknowledges the support of the Ramsay Memorial Fellowship Trust.

\section{APPENDIX A}

For convenience, the relevant spherical Bessel functions are collected here. They are obtained from Eq. (23):

$$
\begin{aligned}
& h_{0}^{(1)}(\kappa R)=-i e^{i \kappa R} / \kappa R, \\
& h_{1}^{(1)}(\kappa R)=-e^{i \kappa R}\left(\frac{1}{\kappa R}+\frac{i}{\kappa^{2} R^{2}}\right), \\
& h_{2}^{(1)}(\kappa R)=e^{i \kappa R}\left(\frac{i}{\kappa R}-\frac{3}{\kappa^{2} R^{2}}-\frac{3 i}{\kappa^{3} R^{3}}\right), \\
& h_{3}^{(1)}(\kappa R)=e^{i \kappa R}\left(\frac{1}{\kappa R}+\frac{2 i}{\kappa^{2} R^{2}}-\frac{15}{\kappa^{3} R^{3}}-\frac{15 i}{\kappa^{4} R^{4}}\right) \text {, } \\
& h_{4}^{(1)}(\kappa R)=e^{i \kappa R}\left(\frac{-i}{\kappa R}+\frac{10}{\kappa^{2} R^{2}}+\frac{45 i}{\kappa^{3} R^{3}}-\frac{105}{\kappa^{4} R^{4}}-\frac{105 i}{\kappa^{5} R^{5}}\right) \text {. }
\end{aligned}
$$

\section{APPENDIX B}

Equations (7) may be obtained explicitly in the impact limit by using 


$$
\begin{aligned}
& \bar{G}_{\nu \mu}(k)=\frac{1}{k-z_{\nu \mu}}, \\
& \bar{G}_{\nu \mu}^{\dagger}(k)=\frac{-1}{k-z_{\nu \mu}^{\dagger}},
\end{aligned}
$$

with $c z_{\nu \mu}^{\dagger} \equiv \omega_{\nu \mu}+\Omega+i \Gamma_{\nu \mu}$, and the relations summarized as

$$
\begin{aligned}
& \frac{1}{\omega \pm i \Gamma}=\frac{\omega \mp i \Gamma}{\omega^{2}+\Gamma^{2}}, \\
& \lim _{\Gamma \rightarrow 0+} \frac{1}{\omega \pm i \Gamma}=P \frac{1}{\omega} \mp i \pi \delta(\omega),
\end{aligned}
$$

which serves as a double-check of Eqs. (7), and compares with Ref. 59.

${ }^{1}$ Th. Förster, in Modern Quantum Chemistry, edited by O. Sinanoglu (Academic, New York, 1965), Vol. III.

${ }^{2}$ V. M. Agranovich and M. D. Galanin, in Electronic Excitation Energy Transfer in Condensed Matter, edited by V. M. Agranovich and A. Maradudin (North-Holland, Amsterdam, 1982).

${ }^{3}$ S. Speiser, Chem. Rev. 96, 1953 (1996).

${ }^{4}$ (a) D. L. Dexter, J. Chem. Phys. 21, 836 (1953); (b) R. E. Merrifield, ibid. 23, 402 (1955); (c) K. R. Naqvi and C. Steel, Chem. Phys. Lett. 6, 29 (1970).

${ }^{5}$ G. D. Scholes and K. P. Ghiggino, in Advances in Multiphoton Processes and Spectroscopy, edited by S. H. Lin, A. A. Villaeys, and Y. Fujimura (World Scientific, Singapore, 1996), Vol. 10, 95-331.

${ }^{6}$ G. D. Scholes and K. P. Ghiggino, J. Phys. Chem. 98, 4580 (1994); note the following corrections in Eqs. (7): Eq. (7e) replace $\left(a b \mid a b^{\prime}\right)$ with $\left(a b \mid a^{\prime} b^{\prime}\right)$, Eq. (7f) replace 2( $\left.a^{\prime} \mid a\right) t$ with $\left(a^{\prime} \mid a^{\prime}\right) t$, and Eq. (7g) replace $3(a a \mid b b) q+\left(a a \mid a^{\prime} b^{\prime}\right)$ with $2(a a \mid b b) q+3\left(a a \mid a^{\prime} b^{\prime}\right)$.

${ }^{7}$ S. H. Lin and W. Z. Xiao, Phys. Rev. E 47, 3698 (1993).

${ }^{8}$ (a) R. Jimenez, S. N. Dikshit, S. E. Bradforth, and G. R. Fleming, J. Phys. Chem. 100, 6825 (1996); (b) S. E. Bradforth, R. Jimenez, F. van Mourik, R. van Grondelle, and G. R. Fleming, ibid. 99, 16179 (1995).

${ }^{9}$ L. Valkunas, E. Akesson, T. Pullerits, and V. Sundström, Biophys. J. 70, 2373 (1996).

${ }^{10}$ K. P. Ghiggino and T. A. Smith, Prog. React. Kinet. 18, 375 (1993).

${ }^{11}$ G. D. Scholes, K. P. Ghiggino, A. M. Oliver, and M. N. Paddon-Row, J. Phys. Chem. 97, 11871 (1993).

${ }^{12}$ (a) R. D. Harcourt, G. D. Scholes, and K. P. Ghiggino, J. Chem. Phys. 101, 10521 (1994); (b) G. D. Scholes, R. D. Harcourt, and K. P. Ghiggino, ibid. 102, 9574 (1994); (c) G. D. Scholes and R. D. Harcourt, ibid. 104, 5054 (1996); (d) R. D. Harcourt, K. P. Ghiggino, G. D. Scholes, and S. Speiser, ibid. 105, 1897 (1996).

${ }^{13}$ G. D. Scholes, A. H. A. Clayton, and K. P. Ghiggino, J. Chem. Phys. 97, 7405 (1992).

${ }^{14}$ J. S. Avery, Proc. Phys. Soc. London 88, 1 (1966).

${ }^{15}$ L. Gomberoff and E. A. Power, Proc. Phys. Soc. London 88, 281 (1966).

${ }^{16}$ D. P. Craig and T. Thirunamachandran, Molecular Quantum Electrodynamics (Academic, New York, 1984).

${ }^{17}$ D. P. Craig and T. Thirunamachandran, Acc. Chem. Res. 19, 10 (1986).

${ }^{18}$ D. L. Andrews, Chem. Phys. 135, 195 (1989).

${ }^{19}$ D. P. Craig and T. Thirunamachandran, Chem. Phys. 167, 229 (1992).
${ }^{20}$ D. L. Andrews and G. Juzeliunas, J. Chem. Phys. 95, 5513 (1991).

${ }^{21}$ D. L. Andrews and G. Juzeliunas, J. Chem. Phys. 96, 6606 (1992).

${ }^{22}$ D. L. Andrews and P. Allcock, Chem. Phys. 198, 35 (1995).

${ }^{23}$ (a) G. D. Scholes and K. P. Ghiggino, J. Chem. Phys. 101, 1251 (1994), and references therein; (b) H. Fidder, J. Knoester, and D. Wiersma, ibid. 95, 7880 (1991); (c) F. C. Spano and S. Mukamel, ibid. 91, 683 (1989).

${ }^{24}$ (a) T. Joo, Y. Jia, and G. R. Fleming, J. Chem. Phys. 102, 4063 (1995); (b) T. Joo, Y. Jia, J.-Y. Yu, D. Jonas, and G. R. Fleming, J. Phys. Chem. 100, 2399 (1996); (c) W. P. de Boeij, M. S. Pshenichnikov, and D. A. Wiersma, ibid. 100, 11806 (1996).

${ }^{25}$ (a) R. Kubo, in Fluctuation Relaxation and Resonance in Magnetic Systems, edited by D. Ter-Haar (Oliver and Boyd, Edinburgh, 1962); (b) Adv. Chem. Phys. 15, 101 (1969).

${ }^{26}$ S. Mukamel, Phys. Rep. 93, 1 (1982).

${ }^{27}$ S. Mukamel, Principles of Nonlinear Optical Spectroscopy (Oxford University Press, New York, 1995).

${ }^{28}$ G. Juzeliunas and D. L. Andrews, Phys. Rev. B 49, 8751 (1994).

${ }^{29}$ G. Juzeliunas and D. L. Andrews, Phys. Rev. B 50, 13371 (1994).

${ }^{30}$ (a) T. Takagahara, E. Hanamura, and R. Kubo, J. Phys. Soc. Jpn. 43, 802, 811, 1522 (1977); 44, 728, 742 (1978); (b) T. Takagahara and E. Hanamura, Solid State Commun. 32, 1 (1979).

${ }^{31}$ (a) S. Mukamel, J. Chem. Phys. 82, 5398 (1985); (b) J. Sue, Y. J. Yan, and S. Mukamel, ibid. 85, 462 (1986).

${ }^{32}$ S. Mukamel, Phys. Rev. A 28, 3480 (1983).

${ }^{33}$ M. Cho, N. F. Scherer, and G. R. Fleming, J. Chem. Phys. 96, 5618 (1992).

${ }^{34}$ S. Mukamel, Annu. Rev. Phys. Chem. 41, 647 (1990).

${ }^{35}$ (a) J. M. Jean, R. A. Friesner, and G. R. Fleming, J. Chem. Phys. 96, 5827 (1992); (b) J. M. Jean and G. R. Fleming, ibid. 103, 2092 (1995).

${ }^{36}$ S. Mukamel and V. Ruposov, Chem. Phys. Lett. 242, 17 (1995).

${ }^{37}$ G. D. Scholes, J. Phys. Chem. 100, 18731 (1996).

${ }^{38}$ G. D. Scholes, R. D. Harcourt, and G. R. Fleming, J. Phys. Chem. (in press).

${ }^{39}$ R. Zwanzig, Physica 30, 1109 (1964).

${ }^{40}$ V. M. Kenkre and R. S. Knox, Phys. Rev. B 9, 5279 (1974).

${ }^{41}$ G. D. Scholes (unpublished).

${ }^{42}$ D. L. Andrews and B. S. Sherborne, J. Chem. Phys. 86, 4011 (1987).

${ }^{43}$ A. Bittner, M.Sc. thesis, University of East Anglia, 1992.

${ }^{44}$ I. S. Gradshteyn and I. M. Ryzhik, Table of Integrals, Series and Prod$u c t s$, 5th ed., edited by A. Jeffrey (Academic, San Diego, 1994).

${ }^{45}$ D. L. Andrews, Faraday Discuss. 99, 375 (1994).

${ }^{46}$ D. L. Andrews, Spectrochim. Acta 46A, 871 (1990).

${ }^{47}$ A. Salam and T. Thirunamachandran, J. Chem. Phys. 104, 5094 (1996).

${ }^{48}$ (a) A. J. Stone, in Theoretical Models of Chemical Bonding, edited by Z. B. Maksić (Springer, Berlin, 1991), Part 4; (b) A. D. Buckingham, Q. Rev. Chem. Soc. (London) 13, 189 (1959).

${ }^{49}$ J. Jerphagnon, Phys. Rev. B 2, 1091 (1970).

${ }^{50}$ D. L. Andrews and T. Thirunamachandran, J. Chem. Phys. 68, 2941 (1978).

${ }^{51}$ D. L. Andrews and W. A. Ghoul, Phys. Rev. A 25, 2647 (1982).

${ }^{52}$ D. L. Andrews and M. J. Harlow, Phys. Rev. A 29, 2796 (1984).

${ }^{53}$ D. L. Andrews and I. D. Hands, Chem. Phys. 213, 277 (1996).

${ }^{54}$ D. L. Andrews and N. P. Blake, J. Phys. A 22, 49 (1989).

${ }^{55}$ E. A. Power and T. Thirunamachandran, Phys. Rev. A 28, 2671 (1983).

${ }^{56}$ G. Juzeliunas, Chem. Phys. 198, 145 (1995).

${ }^{57}$ D. P. Craig and T. Thirunamachandran, Chem. Phys. 135, 37 (1989).

${ }^{58}$ D. P. Craig and T. Thirunamachandran, Proc. R. Soc. Ser. A 410, 307 (1987).

${ }^{59}$ G. D. Scholes and K. P. Ghiggino, J. Chem. Phys. 103, 8873 (1995).

${ }^{60}$ G. D. Scholes and G. R. Fleming (in preparation). 